\title{
Hemodinâmica sistêmica e função ventricular esquerda de ratos hipertensos tornados diabéticos
}

\author{
Systemic hemodynamic and left ventricular function \\ of diabetic-induced hypertensive rats
}

Mário Luís Ribeiro Cesaretti',2, Milton Ginoza', Artur Beltrame Ribeiro', Osvaldo Kohlmann Jr. ${ }^{1}$

1 Disciplina de Nefrologia, Universidade Federal de São Paulo (Unifesp), São Paulo, SP, Brasil ${ }^{2}$ Departamento de Ciências Fisiológicas, Pontifícia Universidade Católica de São Paulo (PUCSP), São Paulo, SP, Brasil
Correspondência para: Osvaldo Kohlmann Junior Disciplina de Nefrologia, Universidade Federal de São Paulo Rua Botucatu, 740

04023-900 - São Paulo, SP, Brasil kohlmann@hrim.com.br.

Recebido em 22/Jan/2010 Aceito em 22/Out/2010

\section{RESUMO}

Objetivo: Avaliar a indução do diabetes melito tipo 1 (DM1) na hemodinâmica sistêmica e função ventricular de ratos normotensos e hipertensos. Materiais e métodos: O DM1 foi induzido por estreptozotocina em ratos Wistar (WST), borderline hypertensive rats (BHR) e spontaneously hypertensive rats (SHR). A hemodinâmica sistêmica foi avaliada por termodiluição e a função ventricular, pela preparação de Langendorff. Resultados: A indução de DM1 produziu aumento na pressão arterial de WST e BHR. O DM1 determinou aumento na resistência periférica total no grupo WST e diminuição do débito cardíaco e do volume sistólico nos grupos WST e BHR. Índices de função sistólica foram reduzidos e a rigidez ventricular, apenas nos ratos WST diabéticos. Todos esses efeitos foram mais proeminentes nos ratos WST diabéticos. Conclusão: O DM1 foi acompanhado por importantes alterações nas funções sistólica e diastólica, levando a uma diminuição nos valores hemodinâmicos sistêmicos que não foram alterados pela hipertensão arterial. Arq Bras Endocrinol Metab. 2010;54(9):842-51

\section{Descritores}

Diabetes melito experimental; hipertrofia ventricular esquerda; função ventricular esquerda; hemodinâmica

\section{ABSTRACT}

Objective: To analyze the effects of type-1 diabetes mellitus (DM1) induction on systemic hemodynamic and ventricular function of normotensive and hypertensive rats. Materials and methods: DM1 was induced by streptozotocin in Wistar rats (WST), borderline hypertensive rats (BHR) and spontaneously hypertensive rats (SHR). The systemic hemodynamic was evaluated by thermodilution and ventricular function by Langendorff preparation. Results: DM1-induction increased tail arterial pressure of WST and BHR. DM1 also increased total peripheral resistance in WST and decrease in cardiac output stroke volume in WST and BHR. Systolic function indexes were reduced and ventricular stiffness increased in all WST-diabetic rats. All of these effects were more prominent on diabetic WST rats. Conclusion: The DM1 in rats was accompanied by important changes in both systolic and diastolic heart function leading to significant changes in the systemic hemodynamics that were not significantly enhanced by hypertension. Arq Bras Endocrinol Metab. 2010;54(9):842-51

Keywords

Diabetes mellitus, experimental; hypertrophy, left ventricular; ventricular function, left; hemodynamics

\section{INTRODUÇÃO}

Z'bem conhecido na literatura, por meio de vários estuCdos epidemiológicos $(1,2)$, que o diabetes melito aumenta as taxas de mortalidade cardiovascular. O estudo de Fein evidenciou que o risco de pacientes diabéticos virem a desenvolver insuficiência cardíaca foi de duas a cinco vezes maior que indivíduos não diabéticos (3). A razão para essa observação é o desenvolvimento de uma miocardiopatia específica secundária ao diabetes melito e que é independente da presença de arteriosclerose e/ou doença hipertensiva (4). 
A miocardiopatia diabética foi descrita por Rubler e cols. (5) em autópsias de pacientes diabéticos que tiveram insuficiência cardíaca sem evidências de doença cardíaca valvular, alcoólica, aterosclerótica e hipertensiva.

Muitos pesquisadores também evidenciam o desenvolvimento de miocardiopatia diabética em animais nos quais o diabetes melito foi induzido por agentes que determinam a destruição das células betapancreáticas. Nesses trabalhos experimentais também se identificaram complicações advindas do diabetes melito, como a nefropatia diabética e a hipertensão arterial $(6,7)$.

Existe uma interação sinérgica entre diabetes melito e hipertensão arterial no desenvolvimento e progressão da nefropatia diabética. De fato, quanto maiores os níveis de hipertensão arterial, maiores serão os graus de desenvolvimento da proteinúria e, sobretudo, a velocidade de declínio da função renal de seres humanos e ratos com diabetes insulinodependentes (6-8).

Por outro lado, quando se observam o desenvolvimento e a progressão da miocardiopatia diabética, a interação entre hipertensão arterial e diminuição da função ventricular parece ser pequena ou às vezes ausente. Em estudo de Rodrigues e McNeill (9), que analisou esse parâmetro em ratos Wistar e espontaneamente hipertensos, tanto a hipertensão arterial quanto a indução de diabetes melito produziram diminuição desse parâmetro a níveis semelhantes, não havendo uma associação sinérgica entre esses dois parâmetros (9).

Portanto, o objetivo deste estudo foi avaliar a interação entre a hipertensão arterial e o diabetes melito no desenvolvimento de miocardiopatia diabética em diferentes estados hipertensivos de ratos portadores de hipertensão genética (ratos espontaneamente hipertensos e ratos com hipertensão borderline) nos quais o diabetes melito tipo 1 foi induzido com estreptozotocina.

\section{MATERIAIS E MÉTODOS}

Foram estudados ratos, com 3 meses de vida, das cepas: Wistar normotenso (WST, $\mathrm{n}=4 \mathrm{l}$ ), borderline hypertensive rats (BHR, $\mathrm{n}=40, \mathrm{Fl}$ do cruzamento dos ratos SHR e fêmea Wistar-Kyoto) e spontaneously hypertensive rats $(\mathrm{SHR}, \mathrm{n}=39)$. Os ratos Wistar foram utilizados em detrimento dos ratos Wistar-Kyoto (WKY, controle natural dos ratos SHR) pelo fato de que estes últimos são moderadamente hipertensos, metabolicamente heterogêneos e podem apresentar algum grau de hipertrofia ventricular esquerda (10-12). Esse protocolo foi aprovado pela Comissão de Ética da Universidade Federal de São Paulo (Unifesp).
Os animais foram acondicionados em gaiolas contendo no máximo cinco animais e mantidos no biotério da disciplina de Nefrologia da Unifesp em temperatura constante, ciclo claro/escuro $(12 \mathrm{~h} / 12 \mathrm{~h})$ e tinham acesso livre à ração-padrão e água potável. Todos os animais foram cedidos pelo Biotério Central da Unifesp. As colônias dos ratos SHR e WKY são derivadas do National Institute of Health (NIH) e catalogadas no International Index of Laboratory Animals.

\section{Indução de diabetes melito tipo 1}

O diabetes foi induzido em 24 ratos Wistar (WST + $\mathrm{DM})$, em 21 ratos BHR (BHR + DM) e 20 ratos SHR $(\mathrm{SHR}+\mathrm{DM})$, por meio de uma injeção única de estreptozotocina (Sigma Chemical, $50 \mathrm{mg} / \mathrm{kg}$ nos ratos $\mathrm{WST}+\mathrm{DM}$ e BHR + DM e $45 \mathrm{mg} / \mathrm{kg}$ nos ratos SHR + $\mathrm{DM})$ na veia caudal. O veículo para administração de estreptozotocina foi $0,1 \mathrm{~mL}$ de tampão citrato. Os animais estavam alimentados quando receberam a injeção de estreptozotocina e durante as 48 horas subsequentes à injeção tiveram trocada a água do bebedouro por solução de glicose a 1,25\%. A menor dose de estreptozotocina administrada aos ratos SHR + DM deveu-se ao fato de que a dose de $50 \mathrm{mg} / \mathrm{kg}$ produziu alta mortalidade nesses animais. Os animais controles (WST, BHR e SHR) foram injetados com o mesmo volume de tampão citrato pela veia da cauda. A glicemia foi verificada três dias após a injeção de estreptozotocina e também ao final da $12^{a}$ semana do estudo. Para a determinação da glicemia, uma gota de sangue foi coletada da veia caudal e colocada em tiras reagentes para mensuração em um glicosímetro (Accutend, Roche). Os animais foram considerados diabéticos se sua glicemia fosse superior a $200 \mathrm{mg} / \mathrm{dL}$. Não foi administrada nenhuma insulina durante o estudo.

Todos os animais foram acompanhados por $12 \mathrm{se}$ manas, e durante esse período a pressão arterial de cauda, por meio do método oscilométrico, e o peso corporal foram verificados duas vezes por semana. Na semana que precedia a administração de estreptozotocina e nas semanas 4,8 e 12 após a indução do diabetes, foi coletada a urina de 24 horas em gaiola metabólica (Nalgene). Na urina de 24 horas foi determinada a albuminúria, um índice de nefropatia diabética, pela técnica de imunodifusão radial.

\section{Estudo hemodinâmico}

Foi separada para o estudo hemodinâmico parte dos animais de cada um dos grupos experimentais. Para 
tanto, os animais foram anestesiados com pentobarbital sódico na dose de $50 \mathrm{mg} / \mathrm{kg} /$ intraperitoneal. O débito cardíaco, a pressão arterial média e a frequência cardíaca foram determinados utilizando o aparelho Cardiomax II (Columbus Inc.). Para essas determinações, cateteres de polietileno (PE-50) foram inseridos na artéria femoral (para determinação da pressão arterial e da frequência cardíaca), no átrio direito, via veia jugular direita (para administração de soro fisiológico gelado a $4^{\circ} \mathrm{C}$ ), e na artéria aorta ascendente, através da artéria carótida esquerda (para colocação do termístor de termodiluição). O tempo total de implantação de todos os cateteres não excedeu 20 minutos. Aguardou-se um período de estabilização de 30 minutos antes de realizar a primeira medida e foram realizadas três a quatro determinações dos parâmetros hemodinâmicos. O Cardiomax II fornece leituras diretas de débito cardíaco, volume sistólico, frequência cardíaca e pressão arterial média. $\mathrm{O}$ único valor calculado foi a resistência periférica total obtida por meio da divisão da pressão arterial média pelo débito cardíaco.

\section{Estudo da função ventricular esquerda}

Os animais restantes foram submetidos ao estudo da função cardíaca, também sob anestesia por pentobarbital sódio na dose de $50 \mathrm{mg} / \mathrm{kg} /$ intraperitoneal. Para avaliar a função ventricular esquerda, utilizou-se a preparação de coração isolado de Langendorff. Trinta minutos antes de o coração ser removido, todos os animais receberam por via intraperitoneal 500 UI de heparina sódica. O líquido de perfusão utilizado foi o tampão de Krebs-Henseleit modificado com glicose e manitol continuamente borbulhado com $95 \% \mathrm{O}_{2}$ e $5 \%$ $\mathrm{CO}_{2}$. Todos os corações foram perfundidos na pressão fixa de $80 \mathrm{mmHg}$ e sob temperatura constante de $37^{\circ} \mathrm{C}$ (Composição do tampão Krebs-Henseleit em mM: 120 $\mathrm{NaCl} ; 5,4 \mathrm{KCl} ; 2,5 \mathrm{MgSO}_{4} ; 1,25 \mathrm{CaCl}_{2} ; 2.0 \mathrm{NaH}_{2} \mathrm{PO}_{4}$; $27 \mathrm{NaHCO}_{3}$; 11 glucose; 4 manitol, mais $60 \mathrm{mg}$ de EDTA e 20 unidades de insulina. $\mathrm{O}$ pH é acertado para $7,4)$.

A performance ventricular foi medida por meio de um balão de látex inserido no interior do ventrículo esquerdo, através do átrio esquerdo e da valva mitral. O balão estava conectado a um cateter de duplo lúmen. A uma das vias do cateter de duplo lúmen estava conectado um transdutor de pressão $(\mathrm{P} 23 \mathrm{~dB}$, Gould Instruments) e a outra, uma seringa Hamilton de 250 microlitros. Após o período de estabilização de 40 minutos, o volume do balão foi ajustado de modo que a pressão diastólica intraventricular esquerda fosse de $0 \mathrm{mmHg}$. $\mathrm{O}$ volume contido dentro do balão, que determinou a pressão diastólica ventricular de $0 \mathrm{mmHg}$, foi chamado de volume inicial $\left(\mathrm{V}_{0}\right)$. O protocolo experimental constituiu da construção de uma curva pressão-volume, em que, através de uma das vias do cateter de duplo lúmen, se colocaram volumes crescentes de 10 microlitros no interior do balão. A cada incremento de volume, aguardavam-se 2 minutos para estabilização e então se procedia ao registro das pressões intraventriculares. Essa manobra findou-se quando a pressão diastólica de 60 $\mathrm{mmHg}$ foi atingida. As variáveis da função sistólica e diastólica ventricular foram registradas em um polígrafo da marca Gould. Para analisar as capacidades contráteis do ventrículo esquerdo, foram utilizadas as medidas da máxima pressão desenvolvida (PDmáx) (diferença entre a pressão sistólica e diastólica) pelo ventrículo esquerdo e a derivada positiva de pressão ventricular $(+\mathrm{dP} /$ dt). A derivada negativa da pressão ventricular $(-\mathrm{dP} / \mathrm{dt})$ foi estudada para avaliar o relaxamento ventricular, e as tangentes das relações pressão-volume em $25 \mathrm{mmHg}$ foram utilizadas para verificar as propriedades passivas do ventrículos (complacência ventricular) (13).

\section{Morfologia ventricular}

Depois de terminados os estudos de hemodinâmica sistêmica e de função ventricular esquerda, todos os ventrículos foram excisados, lavados com salina, secos em papel absorvente e pesados em balança analítica. Foi calculado o peso ventricular esquerdo relativo $(\mathrm{mg} / \mathrm{g}$ de animal), obtido pela divisão do peso ventricular esquerdo absoluto corrigido pelo peso corporal. O peso ventricular esquerdo também foi corrigido pela tíbia esquerda, uma vez que os animais diabéticos tiveram uma grande perda de massa corporal (g/cm de tíbia). Após a pesagem, os corações foram estocados em formol tamponado.

A análise qualitativa do colágeno ventricular foi realizada mediante a fixação dos corações em parafina. Os ventrículos foram cortados no sentido perpendicular (cortes de $2 \mathrm{~mm}$ ) no nível médio dos músculos papilares. Os cortes foram corados com hematoxilina e eosina. A análise qualitativa do conteúdo de colágeno foi feita pela determinação do percentual do número de campos (\%) que continham fibrose perivascular e fibrose intersticial. Foram computados 40 campos aleatórios de cada lâmina.

Os resultados estão apresentados na forma de média \pm erro-padrão da média. A análise de variância de 
medidas repetidas foi utilizada para comparar os valores basais da pressão arterial de cauda e o peso corporal daqueles obtidos nas 12 semanas de acompanhamento. Para comparar a variação temporal da albuminúria, foi utilizada a análise de variância em postos (ANOVA on ranks). A comparação dos vários parâmetros entre os ratos não diabéticos e diabéticos foi feita por meio da análise de variância de uma via e do teste de Kruskal-Wallis. Quando aplicado, a comparação dos ratos diabéticos foi realizada por meio dos testes " $\mathrm{t}$ ” de Student, para os dados paramétricos, e de Mann-Whitney, para os dados não paramétricos. Foram considerados significantes os valores de $\mathrm{p}<0,05$.

\section{RESULTADOS}

Relativamente a pressão arterial, peso corporal, glicemia e albuminúria, os dados estão resumidos nas tabelas 1 e 2.

Tabela 1. Peso corporal (PCorp, gramas), glicemia de jejum (12h) (mg/ $\mathrm{dL}$ ) e excreção urinária de albumina na $12^{\mathrm{a}}$ semana do estudo (EUA, $\mathrm{mg} / 24 \mathrm{~h})$

\begin{tabular}{lccc}
\hline & PCorp & Glicemia & EUA \\
\hline WST $(n=24)$ & $367,8 \pm 8,3$ & $94,3 \pm 1,8$ & $0,43 \pm 0,01$ \\
WST-DM $(n=17)$ & $231,1 \pm 5,3^{\sharp}$ & $335,4 \pm 10,2^{*}$ & $0,86 \pm 0,02^{*}$ \\
BHR $(n=21)$ & $353,8 \pm 3,3$ & $93,1 \pm 0,8$ & $0,79 \pm 0,05^{*}$ \\
BHR-DM $(n=21)$ & $314,4 \pm 14,1^{\sharp}$ & $366,1 \pm 10,1^{*}$ & $3,06 \pm 0,49^{\star * \&}$ \\
SHR $(n=19)$ & $331,1 \pm 4,9$ & $95,7 \pm 1,9$ & $0,99 \pm 0,03^{*}$ \\
SHR-DM $(n=20)$ & $252,7 \pm 9,4^{*}$ & $343,9 \pm 11,1^{*}$ & $4,89 \pm 0,33^{\star * \&}$ \\
\hline
\end{tabular}

*: $p<0,05$ vs. WST; $\&: p<0,05$ vs. WST-DM; : : $p<0,05$ vs. controle não diabético. Média \pm EPM.

Tabela 2. Pressão arterial de cauda, medida em $\mathrm{mmHg}$, no período basal, na $4^{\mathrm{a}}, 8^{\mathrm{a}}$ e $12^{\mathrm{a}}$ semana de estudo dos diferentes grupos

\begin{tabular}{lcccc}
\hline & Basal & $\mathbf{4}^{\text {a }}$ semana & $\mathbf{8}^{\text {a }}$ semana & $\mathbf{1 2}^{\text {a }}$ semana \\
\hline WST $(n=14)$ & $116,1 \pm 5,3$ & $119,3 \pm 4,2$ & $117,0 \pm 4,8$ & $120,5 \pm 5,7$ \\
WST-DM $(n=8)$ & $122,3 \pm 6,7$ & $144,1 \pm 13,2^{* \sharp}$ & $145,2 \pm 8,5^{\star \star}$ & $154,5 \pm 11,0^{\star \star}$ \\
BHR $(n=12)$ & $157,8 \pm 9,2$ & $155,1 \pm 6,9$ & $156,2 \pm 9,6$ & $157,4 \pm 11,6$ \\
BHR-DM $(n=14)$ & $152,4 \pm 7,5$ & $166,2 \pm 13,5^{* \sharp}$ & $171,8 \pm 17,1^{* *}$ & $177,0 \pm 13,6^{* *}$ \\
SHR $(n=10)$ & $195,3 \pm 15,4$ & $195,7 \pm 10,8$ & $202,5 \pm 19,7$ & $206,8 \pm 18,7$ \\
SHR-DM $(n=8)$ & $194,2 \pm 11,9$ & $218,2 \pm 9,5^{*}$ & $216,0 \pm 13,7^{\sharp}$ & $205,2 \pm 20,5$ \\
\hline
\end{tabular}

*: $p<0,05$ vs. período basal; *: $p<0,05$ vs. controle não diabético. Média \pm EPM.

Os pesos corporais dos animais diabéticos foram significantemente menores que o dos animais não diabéticos. Quando as pressões arteriais de cauda dos ratos diabéticos foram comparadas, verificou-se que a indução de diabetes elevou significantemente a pressão arterial de cauda dos ratos Wistar e BHR. A pressão arterial de cauda dos ratos SHR tornados diabéticos não diferiu da dos ratos SHR controles.
A glicemia foi semelhante e significativamente elevada nos três grupos diabéticos estudados quando comparados com os grupos controles não diabéticos, que também não diferiram entre si.

A excreção urinária de albumina foi significativamente maior nos ratos hipertensos (BHR e SHR) quando comparados aos ratos normotensos. A indução de diabetes determinou aumento da albuminúria, proporcional ao grau de hipertensão arterial, em todos os grupos estudados.

\section{Dados hemodinâmicos}

A figura 1 apresenta os dados hemodinâmicos de todos os grupos experimentais. O débito cardíaco (Figura 1A) revelou uma diminuição significante desse parâmetro nos ratos SHR não diabéticos $(197,5 \pm 16,1 \mathrm{~mL} /$ min) em comparação com ratos Wistar e BHR não diabéticos $(257,0 \pm 8,3 \mathrm{~mL} / \mathrm{min}$ e $235,2 \pm 5,3 \mathrm{~mL} / \mathrm{min}$, respectivamente). A indução de diabetes reduziu significantemente o débito cardíaco nas três cepas estudadas a valores que não foram diferentes entre si (WST - DM $=166,1 \pm 6,9 ; \mathrm{BHR}+\mathrm{DM}=184,1 \pm 9,3 \mathrm{e} \mathrm{SHR}+\mathrm{DM}$ $=161,2 \pm 7,2 \mathrm{~mL} / \mathrm{min})$.

Como não existiu diferença entre as frequências cardíacas dos diferentes grupos estudados (WST $=326 \pm$ $3 ; \mathrm{WST}+\mathrm{DM}=323 \pm 13 ; \mathrm{BHR}=258 \pm 9 ; \mathrm{BHR}+$ $\mathrm{DM}=281 \pm 15 ; \mathrm{SHR}=337 \pm 22$ e SHR $+\mathrm{DM}=310$ $\pm 21 \mathrm{bpm}, \mathrm{n} . \mathrm{s}$.), as variações do volume sistólico, como observado na figura $1 \mathrm{~B}$, foram semelhantes às observadas com o débito cardíaco, isto é, os volumes sistólicos foram menores nos animais diabéticos em comparação aos não diabéticos, com exceção dos ratos $\mathrm{SHR}+\mathrm{DM}$ $\mathrm{q}\left(\mathrm{WST}=0,87 \pm 0,02 ; \mathrm{WST}+\mathrm{DM}=0,62 \pm 0,05^{*}\right.$; $\mathrm{BHR}=0,84 \pm 0,02 ; \mathrm{BHR}-\mathrm{DM}=0,68 \pm 0,05^{*} ; \mathrm{SHR}$ $=0,58 \pm 0,05$ e SHR $+\mathrm{DM}=0,54 \pm 0,03 \mathrm{~mL} /$ bat, $\mathrm{p}<$ 0,05 vs. Não diabético).

Por outro lado, a resistência periférica total (Figura 1C), quando comparada entre os animais não diabéticos, mostrou-se significantemente maior nos ratos do grupo BHR e SHR (WST $=0,354 \pm 0,056$; BHR $=0,849 \pm 0,075^{*}$ e $\mathrm{SHR}=0,581 \pm 0,134^{*} \mathrm{mmHg} /$ $\mathrm{mL}^{-1} \cdot \min ,{ }^{*} \mathrm{p}<0,05$ vs. WST). A indução de diabetes tipo 1 produziu incremento na resistência periférica dos ratos WST + DM (WST + DM = 0,543 $\pm 0,0718^{*}$ $\mathrm{mmHg} / \mathrm{mL}^{-1} \cdot \mathrm{min},{ }^{*} \mathrm{p}<0,05$ vs. WST $)$. Nos animais dos grupos BHR + DM e SHR + DM, não se verificou aumento da resistência periférica total $(\mathrm{BHR}+\mathrm{DM}=$ $0,684 \pm 0,140$ e SHR $+\mathrm{DM}=0,648 \pm 0,125 \mathrm{mmHg} /$ $\mathrm{mL}^{-1} \cdot \mathrm{min}$, não significante $v s$. WST $\left.+\mathrm{DM}\right)$. Mais ainda, 
o aumento da resistência periférica total verificado nos ratos Wistar diabéticos foi proporcionalmente maior do que aquele observado nos ratos hipertensos.
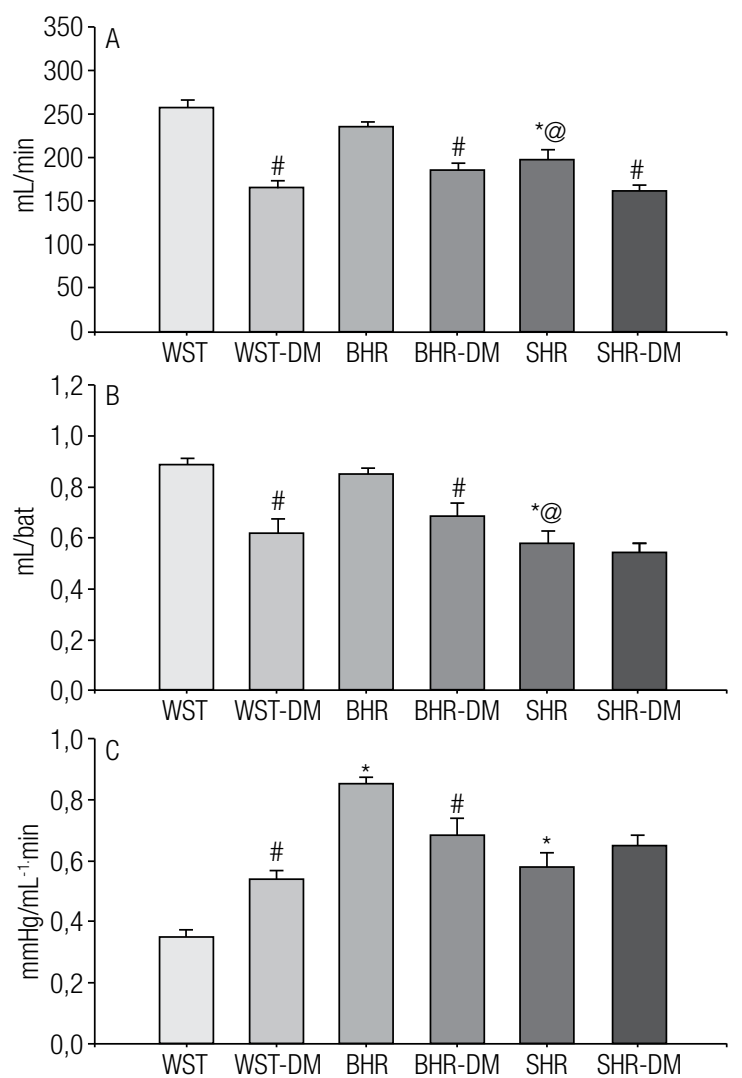

Figura 1. Painel superior: débito cardíaco ( $\mathrm{mL} / \mathrm{min})$; painel intermediário: volume sistólico (mL/bat); painel inferior: resistência periférica total (mmHg/mL $\left.{ }^{-1} \cdot \mathrm{min}\right)$. Resultados: * $\mathrm{p}<0.05 \mathrm{vs}$. WST; ${ }^{\circledR} \mathrm{p}<0.05 \mathrm{vs}$. BHR; ${ }^{\#} \mathrm{p}<0.05$ vs. controles não diabéticos.

\section{Análise da função ventricular}

\section{Função sistólica (Tabela 3)}

Quando comparado aos ratos Wistar não diabéticos, verificou-se que a máxima pressão desenvolvida pelo ventrículo esquerdo $\left(\mathrm{PD}_{\text {Máx }}\right)$ foi significativamente reduzida nos ratos BHR e SHR não diabéticos. A indução de diabetes produziu redução significante desse parâmetro nos ratos WST + DM e BHR + DM, mas não nos ratos $\mathrm{SHR}+\mathrm{DM}$. Os valores de $\mathrm{PD}_{\text {Máx }}$ dos três grupos de ratos diabéticos não apresentaram diferenças entre eles. Mais ainda, o maior grau de redução foi verificado nos ratos WST + DM.

A análise da derivada positiva $(+\mathrm{dP} / \mathrm{dt})$ foi significantemente menor nos ratos hipertensos não diabéticos (BHR e SHR) quando comparados aos controles normotensos (WST). Novamente, o diabetes determinou redução significante $\mathrm{da}+\mathrm{dP} / \mathrm{dt}$ nos ratos $\mathrm{WST}+\mathrm{DM}$ e BHR + DM, mas não nos SHR + DM. Proporcionalmente, houve maior grau de redução $\mathrm{da}+\mathrm{dP} / \mathrm{dt}$ nos ratos WST + DM quando eles foram comparados aos ratos $\mathrm{BHR}+\mathrm{DM}$. Mais uma vez, não houve diferenças entre as $+\mathrm{dP} / \mathrm{dt}$ entre os ratos normotensos e hipertensos tornados diabéticos (Figura 2, painel superior).

\section{Função diastólica (Tabela 3)}

O relaxamento ventricular (-dP/dt, figura 2, painel inferior) em ratos hipertensos não diabéticos (BHR e SHR) foi significativamente menor que em ratos Wistar não diabéticos. A indução de diabetes determinou diminuição significante da derivada negativa, e a maior diminuição desse parâmetro foi verificada no grupo WST + DM. Entretanto, quando se compara o relaxamento ventricular entre todos os grupos diabéticos, verifica-se uma diminuição do relaxamento ventricular proporcional ao grau de hipertensão arterial em todos os grupos diabéticos, evidenciando um sinergismo entre hipertensão arterial e diabetes melito na diminuição da capacidade de relaxamento dos ventrículos esquerdos.

A rigidez ventricular (tangente das relações stress-strain ventriculares, figura 3) estava aumentada nos ratos BHR e SHR não diabéticos quando comparados com os animais Wistar normotensos não diabéticos, porém entre os dois grupos de ratos hipertensos não havia diferença estatística. Quando os animais foram tornados diabéticos, verificou-se um aumento significante na rigidez ventricular apenas nos ratos WST + DM, fato esse que não foi observado nos dois modelos de severidade de hipertensão arterial. Não se encontrou diferença estatística quando se comparou a rigidez ventricular dos ratos BHR e SHR diabéticos ou normoglicêmicos. Os valores da rigidez ventricular dos três grupos de animais diabéticos foram semelhantes.

\section{Morfologia do ventrículo esquerdo (Tabela 3)}

\section{Peso ventricular esquerdo}

Os valores do peso ventricular esquerdo absoluto foram significantemente maiores nos ratos BHR e SHR não diabéticos, quando comparados ao seu controle Wistar. A indução de diabetes diminuiu significativamente o peso ventricular esquerdo absoluto de todos os grupos experimentais $(\mathrm{WST}=0,77 \pm 0,02 ; \mathrm{WST}+\mathrm{DM}=$ $0,59 \pm 0,02 \# ; \mathrm{BHR}=0,96 \pm 0,02 ; \mathrm{BHR}+\mathrm{DM}=0,75$ $\pm 0,03 \#$; SHR $=1,04 \pm 0,02$ e SHR $+\mathrm{DM}=0,76 \pm$ $0,02 \#$ gramas, \#p $<0,05$ vs. não diabético). 
Tabela 3. Máxima pressão desenvolvida pelo ventrículo esquerdo (PDmáx, mmHg), derivada positiva (+dP/dt, mmHg/s), derivada negativa (-dP/dt, $\mathrm{mmHg} / \mathrm{s}$ ), índice de rigidez ventricular (tangente, sem unidade) e peso ventricular esquerdo corrigido pela tíbia (PVE/tíbia, g/cm)

\begin{tabular}{lccccc}
\hline & PDmáx & +dP/dt & -dP/dt & tangente & PVE/tíbia \\
\hline WST $(n=14)$ & $176,4 \pm 12,5$ & $3255 \pm 491$ & $1564 \pm 205$ & $0,196 \pm 0,017$ & $0,197 \pm 0,006$ \\
WST-DM $(n=8)$ & $122,3 \pm 16,8^{*}$ & $1834 \pm 312^{*}$ & $888 \pm 210^{*}$ & $0,330 \pm 0,094^{*}$ & $0,176 \pm 0,010^{*}$ \\
BHR $(n=12)$ & $134,5 \pm 22,6^{*}$ & $2219 \pm 173^{*}$ & $1048 \pm 324^{*}$ & $0,304 \pm 0,098^{\star}$ & $0,273 \pm 0,008^{\star}$ \\
BHR-DM $(n=14)$ & $116,7 \pm 8,4^{*}$ & $1235 \pm 188^{*}$ & $627 \pm 70^{\star *}$ & $0,249 \pm 0,042$ & $0,220 \pm 0,007^{*}$ \\
SHR $(n=10)$ & $113,4 \pm 13,1^{*}$ & $1757 \pm 324^{*}$ & $849 \pm 191^{*}$ & $0,276 \pm 0,051^{*}$ & $0,297 \pm 0,009^{*}$ \\
SHR-DM $(n=8)$ & $112,5 \pm 9,4$ & $1420 \pm 183$ & $532 \pm 206 \&^{*}$ & $0,277 \pm 0,058$ & $0,231 \pm 0,08^{\star *}$ \\
\hline
\end{tabular}

*: $p<0,05$ vs. WST; \&: $p<0,05$ vs. WST-DM; \#: $p<0,05$ vs. controle não diabético. Média \pm EPM.
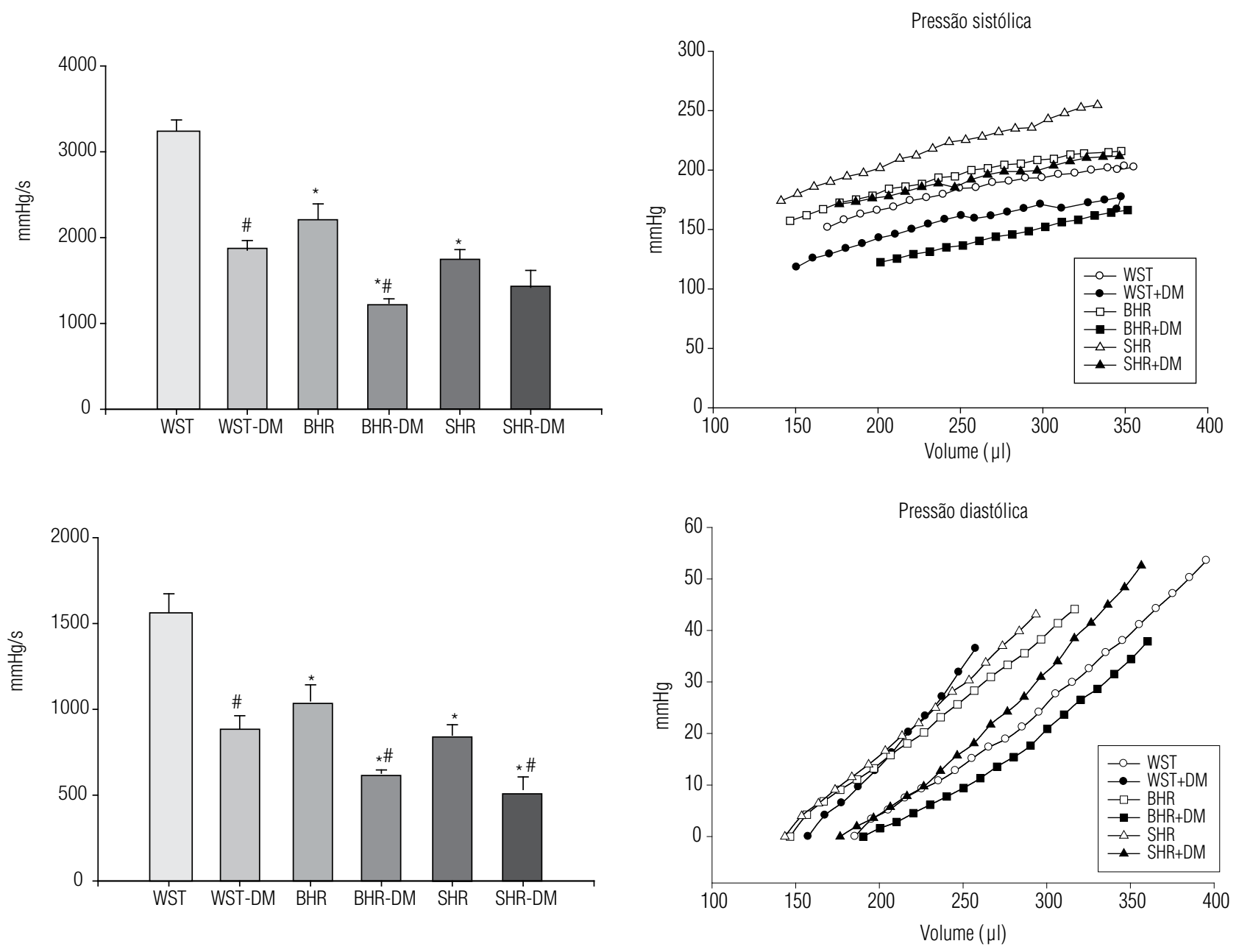

Figura 2. Painel superior: derivada positiva $(+\mathrm{dP} / \mathrm{dt})(\mathrm{mmHg} / \mathrm{s})$; painel inferior: derivada negativa (-dP/dt) (mmHg/s). Resultados: ${ }^{\star} p<0,05 \mathrm{vs}$. WST, ${ }^{\&} p<0,05$ vs. WST + DM, ${ }^{\#} p<0,05$ vs. controle não diabético.

Quando o peso ventricular foi corrigido pelo peso corporal, continuou-se a verificar valores significantemente maiores nos grupos BHR e SHR quando comparados aos ratos WST. Porém, quando se analisa o peso ventricular corrigido pelo peso corporal dos ratos

Figura 3. Curvas pressão-volume (curvas de Starling) sistólica e diastólica dos grupos Wistar (WST), Wistar diabético (WST + DM), BHR, BHR diabético (BHR + DM), SHR e SHR diabético (SHR + DM) obtidas no ventrículo esquerdo durante a preparação de Langendorff.

diabéticos, se verifica um padrão diferente daquele observado na análise do peso ventricular esquerdo absoluto, devido à grande perda de peso dos ratos diabéticos. Nessa situação, o peso ventricular esquerdo relativo aumenta significativamente nos ratos WST + DM, di- 
minui nos ratos $\mathrm{BHR}+\mathrm{DM}$ e não se altera nos ratos SHR + DM. Dessa maneira, fez-se necessário um índice que pudesse expressar de forma fidedigna para correção do peso ventricular. Assim, optou-se por corrigir o peso do ventrículo esquerdo pelo comprimento da tíbia esquerda. Utilizando-se esse índice, verificou-se o mesmo padrão observado quando se realizou a análise do peso ventricular esquerdo absoluto, isto é, aumento da massa ventricular esquerda nos ratos hipertensos e diminuição em todos os ratos diabéticos.

\section{Relação entre o peso ventricular/volume inicial $\left(V_{0}\right)$}

Como demonstrado na literatura $(14,15)$, todos os ventrículos dos animais hipertensos (BHR e SHR) apresentavam um aumento na relação entre o peso ventricular/volume inicial $\left(\mathrm{V}_{0}\right)$ quando comparados aos ratos normotensos (WST) (WST $=4,73 \pm 0,70 ; \mathrm{BHR}$ $=7,16 \pm 2,33^{*} ; \mathrm{SHR}=7,96 \pm 1,12^{*} \mathrm{mg} / \mu \mathrm{l},{ }^{*} \mathrm{p}<0,05$ vs. WST). Quando se compararam esse parâmetro entre ratos normoglicêmicos e diabéticos, verificou-se uma redução desse índice nos ratos $\mathrm{BHR}+\mathrm{DM}$ e SHR + $\mathrm{DM}(\mathrm{WST}+\mathrm{DM}=5,64 \pm 1,06 ; \mathrm{BHR}+\mathrm{DM}=.4,79$ $\pm 1,07 \#, \mathrm{SHR}+\mathrm{DM}=4,99 \pm 0,95 \# \mathrm{mg} / \mu \mathrm{l}, \# \mathrm{p}<0,05$ vs. não diabético).

\section{Análise microscópica}

A análise microscópica qualitativa revelou que os animais BHR e SHR normoglicêmicos apresentavam um maior número de campos contendo lesões intersticiais e perivasculares sugestivas de fibrose (WST $=0,00 \pm 0,0$; $\mathrm{BHR}=25,7 \pm 8,2^{\#} ; \mathrm{SHR}=48,0 \pm 18,0^{\#} \%,{ }^{*} \mathrm{p}<0,05$ vs. WST). Quando se compararam os ratos normoglicêmicos com os diabéticos, houve apenas aumento no número de lesões nos ratos WST + DM (WST + DM = $10,0 \pm 4,2^{*}: \mathrm{BHR}+\mathrm{DM}=30,61 \pm 16,3: \mathrm{SHR}+\mathrm{DM}$ $=36,7 \pm 7,0 \%,{ }^{*} \mathrm{p}<0,05$ vs. não diabético). Também não se verificaram diferenças estatisticamente significantes entre os três grupos diabéticos.

\section{DISCUSSÃO}

\section{Pressão arterial, peso corporal, glicemia e albuminúria}

Os resultados confirmam os dados prévios que mostram que a indução de diabetes melito promove aumento da pressão arterial em ratos normotensos, aumenta o estado hipertensivo de ratos BHR, mas não ganho adicional na pressão arterial de ratos SHR (16-18), e também estão de acordo com a literatura (16) que reportou reduções no peso corporal associado ao desenvolvimento de hiperglicemia. Cabe ressaltar que a hiperglicemia obtida pelos três grupos foi semelhante, apesar de os ratos SHR + DM receberem uma dose menor de estreptozotocina. Essa menor dose deveu-se à grande mortalidade observada nesses ratos quando a dose era de $50 \mathrm{mg} / \mathrm{kg}$. Já é relatado na literatura que nos ratos espontaneamente hipertensos existe uma maior suscetibilidade à administração de estreptozotocina e que menores doses dessa substância produzem efeitos diabetogênicos semelhantes aos de maiores doses quando administradas a ratos Wistar $(19,20)$. A perda de peso em nossos animais é variável e provavelmente se deve às diferentes doses de estreptozotocina utilizadas.

Os resultados deste estudo vão ao encontro de dados prévios deste laboratório (18) e de outros pesquisadores (2l) que demonstram o desenvolvimento da nefropatia diabética, medida pela excreção urinária de albumina, de ratos tornados diabéticos por administração de estreptozotocina. O desenvolvimento da nefropatia diabética, como esperado, foi agravado de maneira dependente da severidade da hipertensão arterial.

\section{Hemodinâmica sistêmica e função cardíaca em ratos diabéticos}

Em nosso estudo, os dados hemodinâmicos dos animais diabéticos foram caracterizados por importante aumento na resistência periférica total associada à diminuição do débito cardíaco e do volume sistólico. Cabe ressaltar, porém, que as medidas hemodinâmicas foram realizadas em animais sob anestesia e o cálculo da resistência periférica foi realizado nessas condições. Os valores da pressão arterial aqui discutidos são dos animais em vigília, obtidos mediante medidas da pressão arterial de cauda. O aumento da resistência periférica nos animais diabéticos e o consequente aumento da pressão arterial de cauda possivelmente se devem à ativação do sistema renina-angiotensina-aldosterona (22) e à disfunção endotelial (23). De fato, estudos com modelo de diabetes induzido por estreptozotocina verificaram também aumento da resistência periférica total $(24,25)$. Mais ainda, nos ratos BHR + DM e SHR + DM, a indução do diabetes pode ter determinado uma maior ativação do sistema nervoso autônomo simpático, uma vez que na etiologia da hipertensão dos ratos BHR e SHR ocorre maior ativação autonômica $(26,27)$. As variações observadas nesses parâmetros foram particularmente maiores 
nos ratos normotensos quando comparados aos ratos hipertensos. Ressalta-se que não houve variação da frequência cardíaca quando se compararam os ratos diabéticos com os não diabéticos.

O maior aumento na resistência periférica total verificada nos ratos WST + DM está de acordo com o maior aumento observado na pressão arterial de cauda, que também sofreu o maior aumento $(+29 \%)$ que nos ratos $\mathrm{BHR}+\mathrm{DM}(+12 \%)$ e SHR $(0 \%)$.

É também interessante notar que a variação percentual dos índices hemodinâmicos foi menor quando se compararam os grupos BHR + DM e SHR + DM com os grupos BHR e SHR, indicando que, apesar de diabéticos, o grau de perda da função foi menor do que aqueles observados nos ratos Wistar tornados. Ressalta-se que os níveis atingidos de débito cardíaco e volume sistólico desses animais hipertensos diabéticos foram semelhantes àqueles obtidos nos ratos WST + DM. Isso se deve ao fato de que ratos não diabéticos BHR e SHR já possuíam valores diminuídos de débito cardíaco e volume sistólico em relação aos ratos não diabéticos WST. Dessa forma, quando ocorreu a interação entre hipertensão arterial e diabetes melito, não houve alterações sobre parâmetros hemodinâmicos maiores que aquelas produzidas isoladamente ou pela hipertensão arterial ou pelo diabetes melito.

As variações hemodinâmicas observadas em nossos grupos provavelmente refletem as modificações nos parâmetros de função sistólica e diastólica pelo diabetes melito. Dessa forma, verificou-se redução significante na contratilidade ventricular esquerda em todos os grupos diabéticos que foram acompanhados por diminuição na máxima pressão ventricular esquerda desenvolvida, indicando uma diminuição da função sistólica. Novamente, as maiores variações na função sistólica foram observadas no grupo WST + DM do que nos hipertensos diabéticos.

A análise da função diastólica evidenciou que houve diminuição da capacidade de relaxamento ventricular, estudado por meio da análise da derivada negativa, verificou-se diminuição no relaxamento ventricular. A indução do diabetes melito produziu redução do relaxamento ventricular, que se associou ao aumento da pressão arterial, isto é, nos grupos $\mathrm{BHR}+\mathrm{DM}$ e SHR + DM, houve maior diminuição do relaxamento ventricular, evidenciando uma interação sinérgica entre pressão arterial e diabetes melito. Os dados referentes à complacência de ratos normotensos e hipertensos normoglicêmicos corroboraram dados prévios da literatura que mostram diminuição da complacência ventricular esquerda de ratos hipertensos (28). Entretanto, quando as propriedades passivas do miocárdio foram comparadas entre os ratos normotensos e hipertensos diabéticos, verificou-se uma dicotomia: uma diminuição da complacência nos ratos WST + DM e ausência de efeitos na complacência de ratos BHR + DM e SHR + DM. A diminuição da complacência pode ser explicada pela quantidade de lesões perivasculares e intersticiais que aumentou apenas nos ratos WST + DM e manteve-se aumentada e inalterada nos ratos hipertensos e hipertensos diabéticos. Uma hipótese possível para que não ocorra maior deposição de colágeno nos ratos BHR + DM e SHR + DM seja que caso haja maior deposição de colágeno estes animais tenha precipitado uma falência miocárdica e estes animais não chegaram ao final do estudo. Destaca-se que, além do próprio diabetes induzido, esses animais já são hipertensos e apresentam certo grau de resistência à insulina (29).

Semelhantemente ao observado na função sistólica, a disfunção diastólica ventricular foi verificada em grau mais intenso nos ratos WST quando tornados diabéticos. Cabe considerar que os animais hipertensos normoglicêmicos já possuíam um grau de diminuição de função diastólica e, dessa forma, o grau de redução da função diastólica nos ratos WST + DM foi proporcionalmente maior que nos ratos hipertensos diabéticos.

Diversos são os subsídios que justificam a disfunção sistólica e diastólica na miocardiopatia diabética nesse modelo experimental. Dentre eles, pode-se destacar o aumento das concentrações intracelulares de ácidos graxos não esterificados (NEFA) $(23,30)$, que repercutem negativamente por determinar abertura de canais de potássio ATP-dependentes $\left(\mathrm{K}_{\mathrm{ATP}}\right)$, diminuindo o período de potencial de ação e o influxo de cálcio no miócito (30). A menor atividade da enzima SERCA-2 (31) determina um déficit de relaxamento do miócito, além de diminuir as atividades de transportadores de membrana como o $\mathrm{Na}^{+}-\mathrm{Ca}^{++}$e o $\mathrm{Na}^{+}-\mathrm{K}^{+}$-ATPase (23). A hiperglicemia observada nos nossos animais, uma vez que eles não recebem insulina, pode determinar alteração na composição de proteínas contráteis do miocárdio (alfa-actina, diminuição da atividade da ATPase da miosina, alem da mudança da isoforma da actina) e produção de compostos de glicolisação avançada que poderiam, respectivamente, determinar diminuição da capacidade contrátil e formação de fibrose, o que contribui para a disfunção diastólica $(30,32)$.

Dessa maneira, como reportado nos dados da hemodinâmica sistêmica, não existe aparente aumento 
pela hipertensão arterial nas mudanças induzidas pelo diabetes nos parâmetros de funções sistólicas e diastólicas, com exceção do grau de relaxamento ventricular, onde se verificou uma relação sinérgica.

Essas observações divergem daquelas descritas por nós e outros pesquisadores que demonstram que o estado hipertensivo acelera o desenvolvimento da nefropatia diabética $(6,21)$. Por outro lado, nossos dados estão de acordo e expandem conceitos de que a associação de diabetes melito e hipertensão arterial não têm efeito aditivo sobre a função cardíaca (29).

Outra possível explicação para o grande déficit produzido nas funções sistólica e diastólica que ocorreram nos ratos WST + DM pode ser o diferente remodelamento ventricular observado nesses ratos. Todos os animais desenvolveram hipertrofia ventricular esquerda quando se corrigiu o peso ventricular esquerdo pelo peso corporal ou pelo comprimento da tíbia. Mais ainda, os ratos hipertensos não diabéticos (BHR e SHR) tiveram um aumento no índice peso ventricular/volume inicial, indicando um remodelamento ventricular concêntrico. Verifica-se o mesmo tipo de remodelamento quando se comparam os ratos normotensos normoglicêmicos e diabéticos, enquanto os ratos hipertensos, quando tornados diabéticos, apresentaram diminuição do índice peso ventricular/volume inicial, indicando hipertrofia ventricular excêntrica.

Em suma, o diabetes induzido por estreptozotocina em ratos foi acompanhado de diminuição importante nas funções sistólica e diastólica, levando a significantes mudanças na hemodinâmica sistêmica. Essas variações não foram significantemente associadas a diferentes graus de hipertensão genética. Nos ratos do grupo Wistar diabético, verifica-se, proporcionalmente, o maior grau de perda nas funções sistólica e diastólica, o que pode ser explicado pela diferente forma de remodelamento ventricular. Esses dados contrastam com os dados de desenvolvimento de nefropatia diabética, que se associa diretamente aos níveis tensionais.

Em conclusão, o diabetes melito experimental em ratos induz miocardiopatia diabética que parece não ser acelerada por diferentes graus de hipertensão arterial.

Declaração: os autores declaram não haver conflitos de interesse científico neste estudo.

\section{REFERÊNCIAS}

1. Kannel WB, Mc Gee DL. Diabetes and cardiovascular disease:The Framingham study. JAMA. 1979;241:2035-8.
2. Torquato MTCG, Montenegro RM, Viana RAHG. Estudo de prevalência do diabetes melito e intolerância à glicose na população urbana de 30 a 69, no município de Ribeirão Preto. Arq Bras Endocrinol Metab. 1999;43:190-9.

3. Fein FS. Diabetic cardiomyopathy. Diabetes Care. 1990;13(11):1169-79.

4. Bell DSH. Diabetic cardiomyopathy: a unique entity or a complication of coronary artery disease. Diabetes Care. 1995;5:708-14.

5. Rubler S, Dlugash J, Yuceoglu YZ, Kumral T, Branwood AW, Grishan A. New type of cardiomyopathy associated with diabetic glomerulosclerosis. Am J Cardiol. 1972;30:595-602.

6. Hasslacher $\mathrm{CH}$, Stech N, Wahl P, Ritz E. Blood factors for nephropathy in type 1 (insulin dependent) diabetes. Diabetologia. 1985;28:6-11.

7. Grossman E, Messerli FH. Diabetic and hypertensive heart disease. Ann Intern Med. 1996;125:304-10.

8. Ribeiro AB. Abnormalities of systemic blood pressure in diabetes mellitus. Kidney Int. 1992;42(6):1470-83.

9. Rodrigues $\mathrm{B}, \mathrm{McNeill} \mathrm{JH}$. Cardiac function in spontaneously hypertensive rats. Am J Physiol. 1986;251:H571-80.

10. Litwin SE, Raya TE, Anderson PG, Daugherty S, Goldman S. Abnormal cardiac function in the streptozotocin-diabetic rat. J Clin Invest. 1990;86:481-8.

11. Pijl AJ, Van der Wal AC, Mathy MJ, Kam KL, Hendriks KL, Pfaffendorf $M$, et al. Streptozotocin-induced diabetes mellitus in spontaneously hypertensive rats: a pathophysiological model for the combined effects of hypertension and diabetes. J Pharmacol Toxicol Methods. 1994;32(4):225-33.

12. Rodrigues B, Cam MC, Kong J, Goyal RK, McNeill JH. Stain differences in susceptibility to streptozotocin-induced diabetes: effects on hypertriglyceridemia and cardiomyopathy. Cardiovasc Res. 1997;33:460-8.

13. Bing OHL, Brooks WW, Robison KG, Slawsky MT, Hayes JA, Litwin $\mathrm{SE}$, et al. The spontaneously hypertensive rat as a model of the transition from compensated left ventriculat hypertrophy to failure. J Moll Cell Cardiol. 1995;27:283-90.

14. De Stefano LM, Matsubara LS, Matsubara BB. Myocardial contractility of the isovolumetrically beating isolated rat heart. Braz $\mathrm{J}$ Med Biol Res. 2004;37(10):1563-9. [cited 2010 Sep 09].

15. Gelsema AJ, Schoemaker RG, Ruzicka M, Copeland NE. Cardiovascular effects of social stress in borderline hypertensive rats. J Hypertens. 1994;12(9):1019-28.

16. Yamamoto J. Blood pressure and metabolic effects of streptozotocin in Wistar-Kyoto and spontaneously hypertensive rats. Clin Exp Hypertens A. 1988;10(6):1065-83.

17. Kusaka M, Kishi K, Sokabe H. Does so-called streptozocin hypertension exist in rats? Hypertension. 1987;10(5):517-21.

18. Ferreira SRG, Zanella MT, Kohlmann Jr O, Mezzomo NF, Ribeiro AB. Hypertension and glucose metabolism disturbance. Prog Pharmacol Clin Plarmacol. 1995; 102:35-40.

19. Jian K, Fok E, Cam MC, Sambandam N, Yao J, Rodrigues B. Susceptibility of spontaneously hypertensive rats to the diabetogenic effects of streptozotocin. Can J Physiol Pharmacol. 1996;74:1215-21.

20. Reaven GM, Hoa H. Low-dose streptozotocin-induced diabetes in the spontaneously hypertensive rat. Metabolism. 1991;40(4):335-7.

21. Mavrakanas TA, Cheva A, Kallaras K, Karkavelas G, MironidouTzouveleki M. Effect of ramipril alone compared to ramipril with eplerenone on diabetic nephropathy in streptozocin-induced diabetic rats. Pharmacology. 2010;86(2):85-91.

22. Poornima IG, Parikh P, Shannon RP. Diabetic cardiomyopathy: the search for a unifying hypothesis. Circ Res. 2006;98(5):596-605.

23. Okoshi K, Guimarães JF, Di Muzio BP, Fernandes AA, Okoshi MP. Miocardiopatia diabética. Arq Bras Endocrinol Metabol. 2007;51(2):160-7.

24. Gurney AM, Howarth FC. Effects of streptozotocin-induced diabetes on the pharmacology of rat conduit and resistance intrapulmonary arteries. Cardiovasc Diabetol. 2009;8:4. 
25. Pugliese G, Pricci F, Barsotti P, lacobini C, Ricci C, Oddi G, et al. Development of diabetic nephropathy in the Milan normotensive strain, but not in the Milan hypertensive strain: possible permissive role of hemodynamics. Kidney Int. 2005;67(4):1440-52.

26. Kunes J, Dobesová Z, Musilová A, ZídekV, Vorlícek J, Pravenec M, et al. Hemodynamic characterization of recombinant inbred strains: twenty years later. Hypertens Res. 2008;31(8):1659-68.

27. DiBona GF, Jones SY. Cardiac volume receptor reflex in borderline hypertensive rats. Hypertension. 1993;21(2):222-6.

28. Cesaretti MLR, Tucci PJF. Reversão da hipertrofia cardíaca. Revista da Sociedade de Cardiologia do Estado de São Paulo. 1994;4:393-400.
29. Riva E, Andreoni G, Bianchi R, Latini G, Jeremic G, Traquandi C, et al. Changes in diastolic function and collagen content in normotensive and hypertensive rats with long term streptozotocin induced diabetes. Pharmacol Res. 1998;37(3):233-40.

30. Boudina S, Abel ED. Diabetic cardiomyopathy, causes and effects. Rev Endocr Metab Disord. 2010;11(1):31-9.

31. Liu GX, Hanley PJ, Ray J, Daut J. Long-chain acyl-coenzyme A esters and fatty acids directly link metabolism to K(ATP) channels in the heart. Circ Res. 2001;88(9):918-24.

32. Wakasaki H, Koya D, Schoen FJ, Jirousek MR, Ways DK, Hoit BD, et al. Targeted overexpression of protein kinase $C$ beta2 isoform in myocardium causes cardiomyopathy. Proc Natl Acad Sci U S A. 1997;94(17):9320-5. 